\title{
Experimental study of needle-tissue interaction forces: effect of needle geometries, insertion methods and tissue characteristics.
}

\author{
Shan Jiang \\ Centre for Advanced Mechanisms and Robotics, School of Mechanical Engineering, Tianjin University, \\ Tianjin, China, 300072
}

\section{Pan Li}

Centre for Advanced Mechanisms and Robotics, School of Mechanical Engineering, Tianjin University, Tianjin, China, 300072

Yan Yu

Division of Medical Physics, Department of Radiation Oncology, Thomas Jefferson University, Kimmel Cancer Center, 111 South 11th Street, Philadelphia, PA 19107, U.S.

Jun Liu

Pepartment of Imaging Tianiin Union.Medicine Center Tianiin, China 300121.

iverngołangoncology Commons

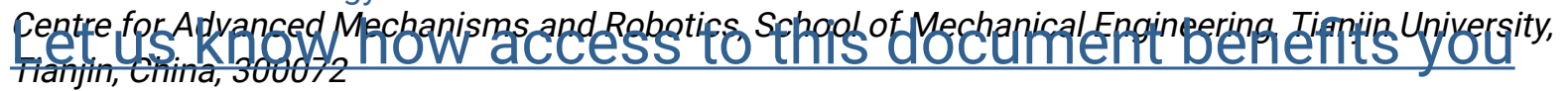

\section{Recommended Citation}

Jiang, Shan; Li, Pan; Yu, Yan; Liu, Jun; and Yang, Zhiyong, "Experimental study of needle-tissue interaction forces: effect of needle geometries, insertion methods and tissue characteristics." (2014). Department of Radiation Oncology Faculty Papers. Paper 48.

https://jdc.jefferson.edu/radoncfp/48

This Article is brought to you for free and open access by the Jefferson Digital Commons. The Jefferson Digital Commons is a service of Thomas Jefferson University's Center for Teaching and Learning (CTL). The Commons is a showcase for Jefferson books and journals, peer-reviewed scholarly publications, unique historical collections from the University archives, and teaching tools. The Jefferson Digital Commons allows researchers and interested readers anywhere in the world to learn about and keep up to date with Jefferson scholarship. This article has been accepted for inclusion in Department of Radiation Oncology Faculty Papers by an authorized administrator of the Jefferson Digital Commons. For more information, please contact: JeffersonDigitalCommons@jefferson.edu. 
As submitted to:

Joumal of biomechanics.

And later published as:

Experimental study of needle-tissue interaction forces: Effect of needle geometries, insertion methods and tissue characteristics

October 2014

Volume 47, Issue, 13, pages: $3344-53$

DOI: 10.1016/j.jbiomech.2014.08.007

Authors: Shan Jiang ${ }^{1}$, Pan $\mathrm{Li}^{1}$, Yan $\mathrm{Yu}^{2}$, Jun Liu ${ }^{3}$, Zhiyong Yang ${ }^{1}$

Affiliations:

1. Centre for Advanced Mechanisms and Robotics, School of Mechanical Engineering, Tianjin University, Tianjin, China, 300072.

2. Division of Medical Physics, Department of Radiation Oncology, Thomas Jefferson University, Kimmel Cancer Center, 111 South 11th Street, Philadelphia, PA 19107, U.S.

3. Department of Imaging, Tianjin Union Medicine Center, Tianjin, China, 300121.

\section{Corresponding author:}

Shan Jiang

School of Mechanical Engineering

Tianjin University

Tianjin, China 300072

Phone: 86-22-27890307 
Fax: 86-22-27890307

Email: shanjmri@tju.edu.cn

Abstract: A thorough understanding of needle-tissue interaction mechanics is necessary to optimize needle design, achieve robotically needle steering, and establish surgical simulation system. It is obvious that the interaction is influenced by numerous variable parameters, which are divided into three categories: needle geometries, insertion methods, and tissue characteristics. A series of experiments are performed to explore the effect of influence factors (material samples $n=5$ for each factor) on insertion force. Data were collected from different biological tissues and a special tissue-equivalent phantom with similar mechanical properties, using a 1-DOF mechanical testing system instrumented with a 6-DOF force/torque (F/T) sensor. The experimental results indicate that three basic phases (deformation, insertion, and extraction phase) are existent during needle penetration. Needle diameter $(0.7 \sim 3.2 \mathrm{~mm})$, needle tip (blunt, diamond, conical, and beveled) and bevel angle $\left(10^{\circ} \sim 85^{\circ}\right)$ are turn out to have a great influence on insertion force, so do the insertion velocity $(0.5 \sim 10 \mathrm{~mm} / \mathrm{s})$, drive mode (robot-assisted and hand-held), and insertion process (interrupted and continuous). Different tissues such as skin, muscle, fat, liver capsule and vessel are proved to generate various force cure, which can contribute to the judgment of needle position and provide efficient insertion strategy.

Keywords: Needle insertion; interaction forces; needle geometry; insertion method; tissue characteristic.

\section{Introduction}

Brachytherapy for lung cancer or liver cancer using surgical tools are common minimally invasive procedures, in which medical needles are widely used (Abolhassani and Patel 2007). The curative effect is highly dependent on the accuracy of penetration (Misra et al. 2010). Therein, tissue deformation and needle deflection that influence the curative effect are affected by the needle-tissue interaction forces 
(DiMaio and Salcudean 2005). The magnitudes of insertion forces and tissue indentation have been investigated considering different parameters before. Thus, a thorough understanding of needle-tissue interaction mechanics is presented, which is beneficial to realistic surgical simulation, preoperative planning and robot-assisted mechanical procedures. Gerwen et al. (2012) provided an extensive overview of experimental insertion-force data available in the literature and investigated the numerous factors that influence the needle-tissue interaction. Okamura et al. (2004) characterized the effects of needle diameter and tip type on insertion force using a silicone rubber phantom. Forces for larger diameter needles are higher due to increased cutting and friction forces. Mahvash et al. (2010) showed that increasing the velocity of needle insertion will reduce the force of rupture event when it increases the energy release rate. To simulate the multilayer insertion during surgery, Yan et al. (2006) performed the extensive experiments in various phantoms, including pure gelatin phantoms and animal tissue phantoms. Lewis et al. (2000) investigated the effect of bevel orientation on force required to puncture human dura using a Tuohy needle. In addition, the needle sharpness, rotating needle insertions (Meltsner et al. 2007), lubrication (Stellman 2009), and decay time (Choy et al. 2002) have been investigated. Nevertheless, a systematic research about influence factors of needle-tissue interaction is still imperative and necessary. Therefore, a comprehensive investigation has been performed on the basis of the experimental data. The numerous variable parameters in the research include needle geometries, insertion methods, and tissue characteristics.

The paper is organized as follows: Section 2 is dedicated to introduce the experimental material, experimental equipment, the needle-tissue interactions model and a statistical analysis method. The analysis of experimental results and discussion are described in Section 3. Finally, we summarize the main contribution of this research and talk about future work in Section 4. 


\section{Materials and Methods}

\subsection{Experimental material}

A transparent Poly (vinyl alcohol) (PVA) hydrogel was adopted as tissue-equivalent material in a series of experiments, especially in tests which investigate needle geometries and insertion methods. The PVA hydrogel with specified formula was prepared by means of physical and chemical cross-linking. It has been demonstrated that when PVA concentration is $8 \mathrm{~g} / \mathrm{dl}$ with a mix water $/ \mathrm{NaCl} /$ DMSO solvent, prepared with 7 freeze/thaw cycles, the material has similar biomechanical characterizations and morphological structure with porcine liver (Jiang and Liu 2011). The force profile acquired from linearly penetration into porcine liver and PVA hydrogel was shown in Fig. 1(a). The test data of PVA hydrogel showed a resemblance to that measured from porcine liver without capsule, which provided evidence that the PVA phantom can be used in ex vivo insertion test with tissue-mimicking properties. Moreover, PVA phantoms can offer a controlled environment for repeatable experiments and a clear observation owing to its transparency. Therefore, at the time of testing, PVA samples ( $\mathrm{n}=5$ for each factor) were sized into $60 * 60 * 60 \mathrm{~mm}^{3}$ cubes using razor blades and were kept in closed containers when not in use to prevent dehydration (Fig. 1(b)). Mean forces can be calculated from data that measured from the 5 tests designed for each factor to eliminate the influence of externalities.

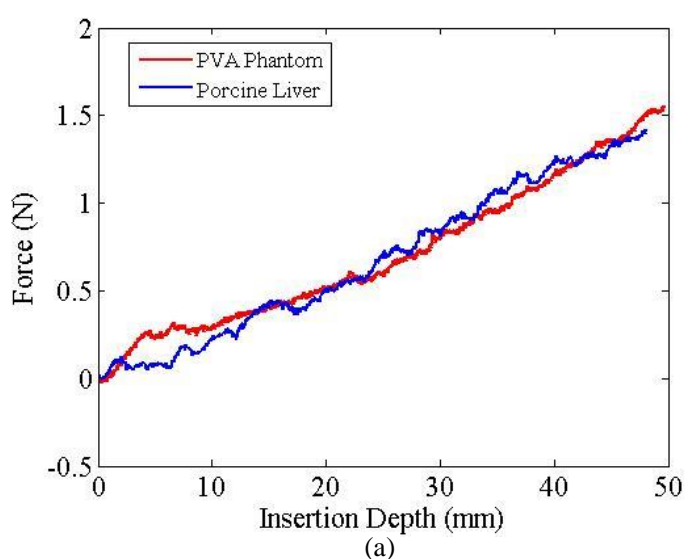

(a)

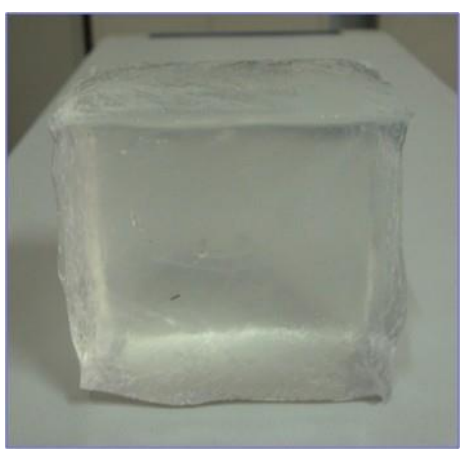

(b) 
Fig. 1. (a) shows the comparison result of experimental interactive forces loaded on porcine liver and on PVA hydrogel, while (b)

is the PVA hydrogel with similar mechanical properties to porcine liver and transparent effect.

\subsection{Experimental equipment}

A 1-DOF mechanical testing system was used for biomechanical testing of needle insertion (Fig.2).

The PVA phantom was placed within a test chamber in front of the horizontal actuator, which allowed for control of sample test locations. A horizontal actuator was equipped with a 6-DOF force/torque (F/T) sensor (Nano-17 from ATI Industrial Automation) to measure the forces and torques acting on the needle and a custom needle mount to hold flexible needles ( $7 \mathrm{G}$, bevel tip) and allow for penetration into the materials. The commercially available puncture needles with different types were adopted in the experiments to discuss the influence of needle geometry during the insertion phases. A servo motor (MINAS A4 from Panasonic Corporation of China) was used to drive needle into phantoms by the lead-screw mechanism at different velocities, and specified insertion velocity during penetration was maintained using a proportional derivative controller for the transverse platform.

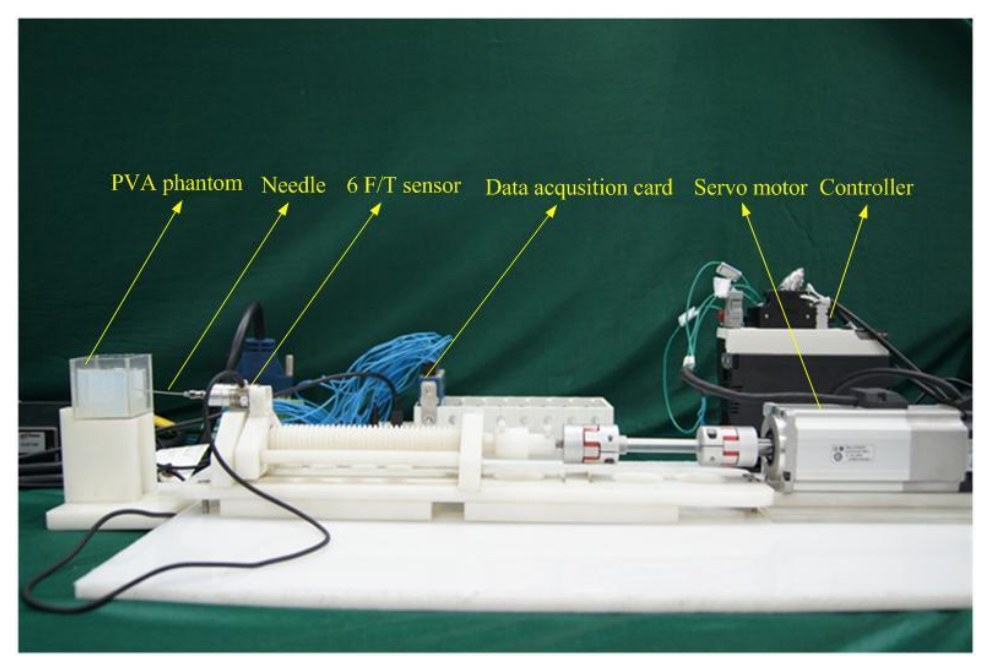

Fig. 2. The experimental setup for data collection.

\subsection{Analysis of needle-tissue interactions model}


In order to build the insertion force model accurately, we make the needle-tissue interaction force divided into three parts: stiffness force, friction force, and cutting force (Okamura et al. 2004).

The stiffness force is due to the skin viscoelasticity or the elastic properties of organ capsule during the puncture in the surface. We employ a contact model (Fig. 3(a)) here that calculates the force-deformation response of a needle in contact with a soft tissue. The contact mechanics problem can be solved from a systematic use of Hankel transforms and the theory of dual integral equation. The elementary solution enables us to derive the expressions (Sneddon et al. 1965):

$$
\begin{gathered}
h=\int_{0}^{1} \frac{f^{\prime}(x)}{\sqrt{1-x^{2}}} d x \\
f_{\text {stiffness }}=2 E_{r} a \int_{0}^{1} \frac{x^{2} f^{\prime}(x)}{\sqrt{1-x^{2}}} d x
\end{gathered}
$$

in which the function $f(x)$ is prescribed by the fact that, referring to the needle tip as origin, the curve of needle has expression $y=f(r)$ where $r=a x(r \leq a)$ so that $f(0)=0 ; a$ is the radius of the circle of contact and $h$ is a parameter whose physical significance is that it is the depth to which the tip of the needle penetrates into the soft tissue; $E_{r}$ represents the reduced modulus that can be determined by the following equation: $\quad \frac{1}{E_{r}}=\frac{1-v_{1}^{2}}{E_{1}}+\frac{1-v_{2}^{2}}{E_{2}}$

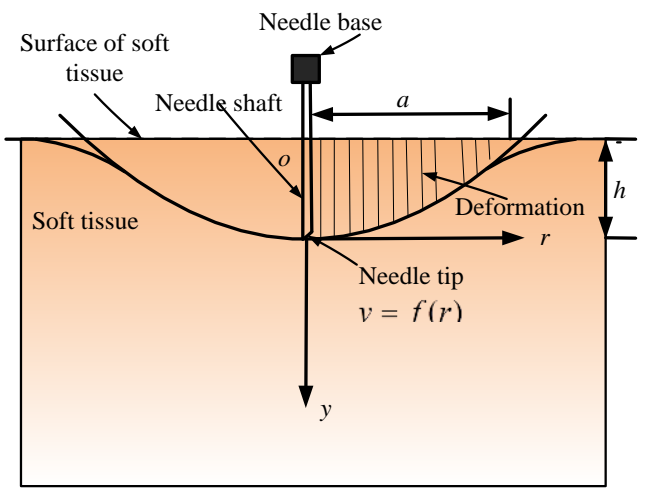

(a)

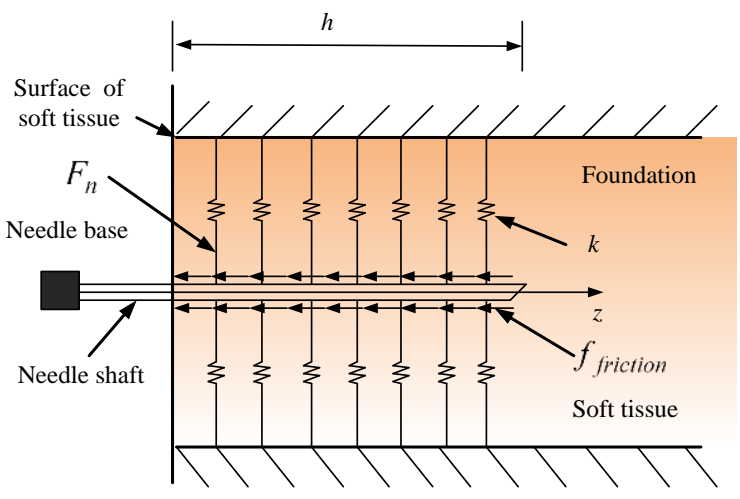

(b)

Fig. 3. The sketch of needle-tissue interaction force model. (a) Contact mechanics model for stiffness force, (b) Modified Winkler's 
where $E_{1}, E_{2}$ and $v_{1}, v_{2}$ are the Young's modulus and the Poisson ratio of needle and soft tissue, respectively. Although the expression (2) is the solution to the axisymmetric Boussinesq problem, the beveled-tip needle is analogous to the conical punch in that they are point contact with the soft tissue. For simplifying the formula of the stiffness force, we take $f(x)=\varepsilon x$ where $\varepsilon=a \cot \alpha$ for normal penetration by a bevel angle $\alpha=15^{\circ}$. Then the function $f(x)$ can be written as:

$$
f(x)=a x \cot \alpha
$$

Substituting the expression (4) and (1) into equation (2), the stiffness force is:

$$
f_{\text {stiffness }}=\frac{2}{\pi} E_{r} \tan (\alpha) h^{2}
$$

Combine Eq. (3) and Eq. (5), a new stiffness force formula is derived:

$$
f_{\text {stiffness }}=\frac{2 E_{1} E_{2}}{\pi\left[E_{2}\left(1-v_{1}^{2}\right)+E_{1}\left(1-v_{2}^{2}\right)\right]} h \cdot \tan (\alpha) \cdot h=\frac{2 E_{1} E_{2}}{\pi\left[E_{2}\left(1-v_{1}^{2}\right)+E_{1}\left(1-v_{2}^{2}\right)\right]} l \cdot h
$$

where $h$ and $l$ represent the axial length and transverse length of bevel tip (Fig. 4), respectively. Since we only change the needle diameter, the Young's modulus and the Poisson ratio of needle and soft tissue remains constant, and then the stiffness force of each needle with different diameter can be reduced to equation as follows:

$$
f_{\text {stiffness }(i)}=\frac{2}{\pi} \psi\left(E_{1}, E_{2}, v_{1}, v_{2}\right) l_{i} \cdot h_{i} \quad\left(\left(0<h_{i} \leq z_{i}, 0<l_{i} \leq d_{i}\right) i=1,2,3,4,5\right)
$$

where $d_{i}$ and $z_{i}$ stand for the diameter of insertion needle (that is, maximum transverse length of bevel tip) and maximum axial length of bevel tip (Fig. 4), respectively. The peak value of stiffness force can be expressed below:

$$
f_{\text {peak }(i)}=\frac{2}{\pi} \psi\left(E_{1}, E_{2}, v_{1}, v_{2}\right) l \cdot h=\frac{2 E_{1} E_{2}}{\pi\left[E_{2}\left(1-v_{1}^{2}\right)+E_{1}\left(1-v_{2}^{2}\right)\right]} d_{i} \cdot z_{i} \quad(i=1,2,3,4,5)
$$

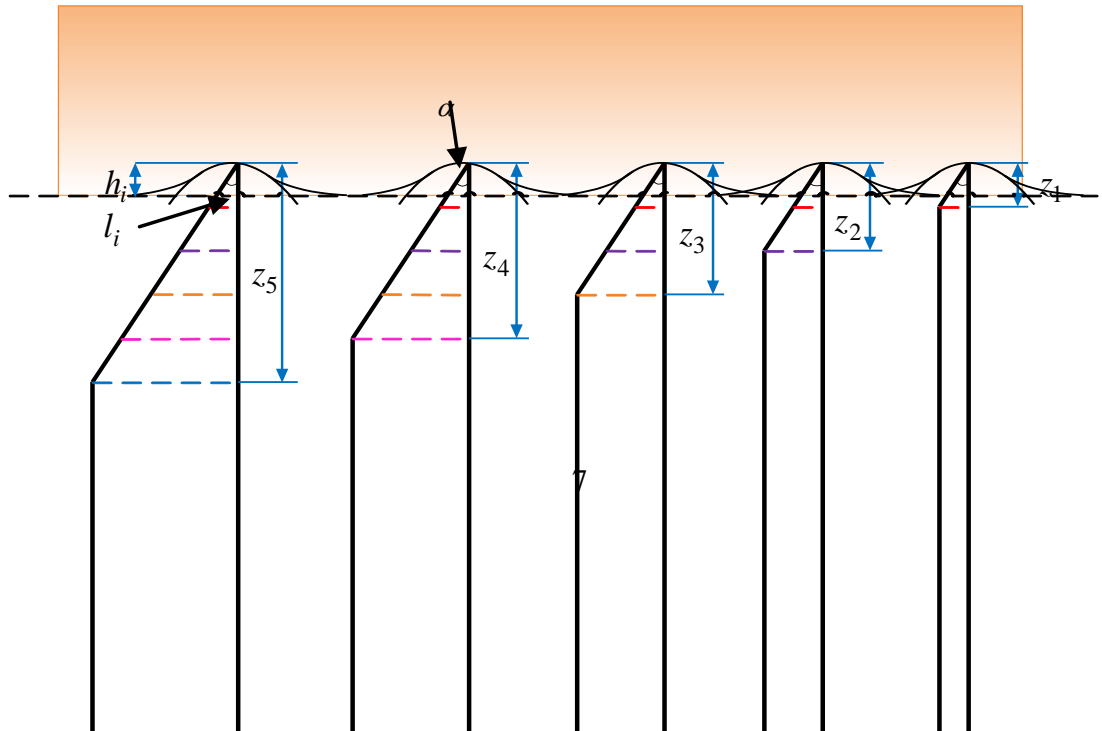


Fig. 4. Penetration into soft tissue using needles with different diameters and the same bevel angle.

The needle force distribution indicates that axial forces between the needle and the tissue phantom are relatively uniform along the needle shaft (DiMaio and Salcudean 2003), so it is reasonable to assume a linear lateral force response for small displacement. Thus, the distributed force along the needle axis could be modeled as a modified Winkler's foundation (Yankelevsky et al. 1989) (Fig. 3(b)) with linear stiffness coefficient:

$$
F_{n}=k h \Delta
$$

where $F_{n}$ is the normal force along the needle shaft due to the tissue deformation, $\Delta$ is the settling amount, $k$ is the foundation modulus and $h$ refers to the contact length.

Biot (1937) has developed the expressions of foundation modulus of the elastic beam $k$ :

$$
k=\frac{\beta E}{\left(1-v_{2}^{2}\right)}\left[\frac{E_{2} b^{4}}{E_{1} I\left(1-v_{2}^{2}\right)}\right]^{\gamma}
$$

where $\beta$ and $\gamma$ are the working condition coefficient. Here we chose $\beta=0.65, \gamma=\frac{1}{12}$, the foundation modulus, $k$, used in needle-tissue interaction has been expressed as:

$$
k=\frac{0.65 E_{2}}{1-v_{2}^{2}} \sqrt[12]{\frac{E_{2} b^{4}}{E_{1} I\left(1-v_{2}^{2}\right)}}
$$

where $I$ and $b$ are moment of inertia of the needle and foundation width, respectively. The friction force acts on the side wall of the needle shaft in the axial direction is viewed as Coulomb friction. Then, the friction force can be expressed as:

$$
f_{\text {friction }}=\mu F_{n}
$$

$\mu$ is the friction coefficient between needle and the soft tissue. Substituting this expression (9), (11) into 
Eq. (12). In this work, the friction is simplified using $\Delta=D / 2$ and $b=\pi D, D$ is the outer diameter of needle. The friction force model can be written as:

$$
f_{\text {friction }}=-\frac{\mu D}{2} \frac{0.65 E_{2}}{1-v_{2}^{2}} \sqrt[12]{\frac{E_{2}(\pi D)^{4}}{E_{1} I\left(1-v_{2}^{2}\right)}} h
$$

Hing et al. (2006), found that the stable cutting force is more or less constant, in porcine liver ex vivo, with some fluctuations due to rupture, depending on the level of inhomogeneity of the tissue. Thus, the cutting force can be expressed as:

$$
f_{\text {cutting }}=C
$$

The complete insertion force model profile is established by summing up equations obtained above.

$$
f_{\text {needle }}(z)= \begin{cases}f_{\text {stiffness }}=\frac{2}{\pi} E_{r} \tan (\alpha) z^{2} & z_{A} \leq z \leq z_{B} \\ f_{\text {friction }}+f_{\text {cutting }}=\frac{\pi \mu D}{2} \frac{0.65 E_{2}}{1-v_{2}^{2}} 12 \sqrt{\frac{E_{2}(\pi D)^{4}}{E_{1} I\left(1-v_{2}^{2}\right)}} z+C & z_{B} \leq z \leq z_{C} \\ f_{\text {friction }}=-\frac{\pi \mu D^{2}}{2} \frac{0.65 E_{2}}{1-v_{2}^{2}} \sqrt[12]{\frac{E_{2}\left(\pi D^{4}\right)}{E_{1} I} z} & z_{C} \leq z \leq z_{D}\end{cases}
$$

Where $z_{A}$ is the position of the undeformed tissue surface, $z_{B}$ is the position of the maximally deformed tissue surface before puncture, $z_{C}$ is the maximal insertion depth, and $z_{D}$ is the position where the needle separates from the soft tissue.

\subsection{Statistical analysis}

Data was analyzed using an analysis of variance (ANOVA) and a standard statistical software package (SPSS 19.0, IBM SPSS, NY, USA). For biomechanical analysis, multiple comparisons were made within levels of independent variables. $p$-values were calculated for a significance level $\alpha=0.05$. The null hypothesis (i.e., equal mean at all factor levels) was used to determine if the mean values of different factors (e.g., needle geometries, tissue characteristics and insertion methods) were statistically different. The null hypothesis was rejected for $p<0.05$.

\section{Results}


During needle penetration, three basic phases of interaction (Fig. 5) are distinguished as follows:

(a) Deformation phase (from A to B): Tissue deformation occurs when the needle comes into contact (A) with the skin or organ capsule until it ruptures, and the insertion force reaches a peak value (B). In this phase, the insertion force is equal to the stiffness force.

(b) Insertion phase (from B to C): The needle penetrates into soft tissue. During insertion phase, the measured insertion force is a summation of friction and cutting force, which is called total force.

(c) Extraction phase (from $\mathrm{C}$ to D): The needle is extracted from the soft tissue. The extraction force is due to the friction force because there is no incision in this phase.
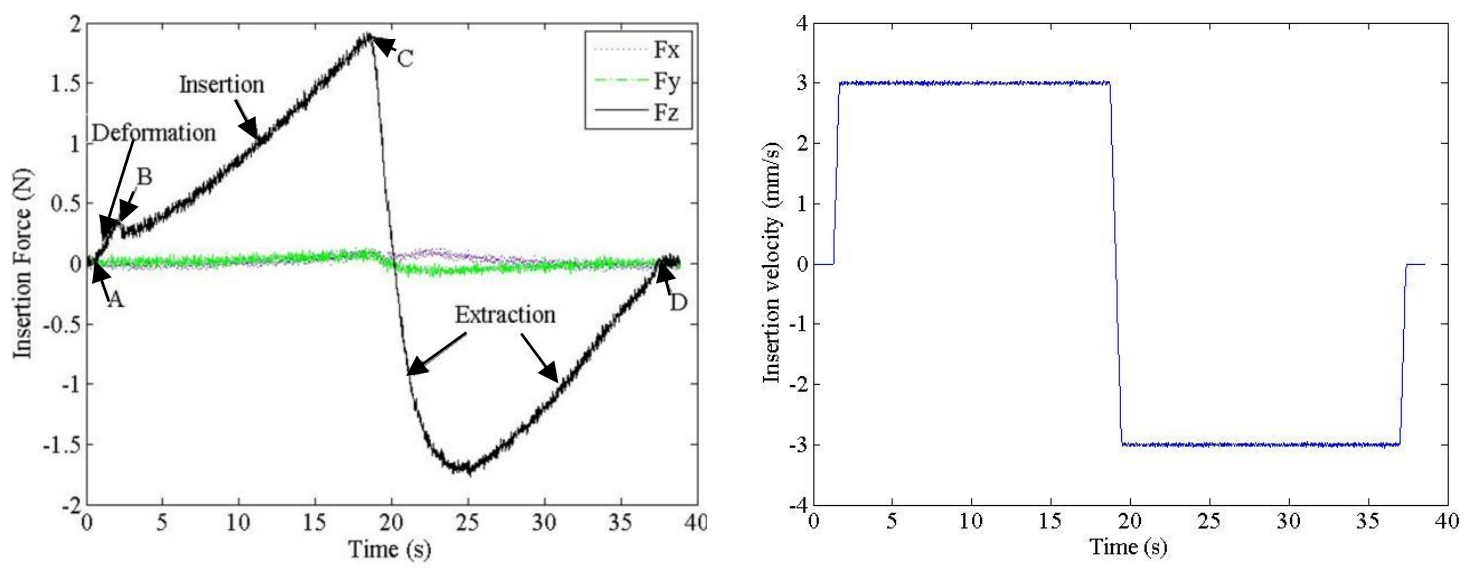

Fig. 5. The force profile of needle-tissue interaction forces at $3 \mathrm{~mm} / \mathrm{s}$.

\subsection{Influence of needle geometry}

To discuss the effect of needle geometries on insertion force, we choose needles with different diameters, tip shapes, and bevel angles. Table 1 lists the tip types, tip angles and diameters. The experiments for each factor are done for 5 times and each test is conducted in different positions of the same PVA phantom, and insertion velocity is constant at $4 \mathrm{~mm} / \mathrm{s}$ in order to avoid the influence of other factors. The insertion needle and force profile is shown in Fig. 6. The stiffness force is found to increase remarkably with diameter (Fig. 6(a), $p \approx 0$ ), and it can be observed that the slope of total force is almost 
parallel to each other.

The stiffness force enlarged with the increasing of bevel angle, which is consistent with Eq. (5), while the total force is found to decrease quickly (Fig. 6(b), $p=3.0834 \mathrm{E}-210, p<0.001$ ). The total force appears periodic fluctuation when the bevel angle is larger than $30^{\circ}$, and the amplitude of the fluctuation is equal to $0.2471 \pm 0.056 \mathrm{~N}$.

\section{Table1}

Diameters, tip types and bevel angles of needles used in experiments, with puncture forces and the slope of axial insertion forces.

\begin{tabular}{cccc} 
& Diameter & Puncture Force & Force density \\
Needle Type & $(\mathrm{mm})$ & $(\mathrm{N})$ & 0.017 \\
\hline $30^{\circ}$ bevel & 0.7 & 0.1456 & 0.021 \\
$30^{\circ}$ bevel & 0.9 & 0.2391 & 0.022 \\
$30^{\circ}$ bevel & 1.2 & 0.3087 & 0.025 \\
$30^{\circ}$ bevel & 1.8 & 0.5441 & 0.026 \\
$30^{\circ}$ bevel & 3.2 & 1.4542 & 0.053 \\
$30^{\circ}$ Cone & 0.7 & 0.2892 & 0.045 \\
$30^{\circ}$ diamond & 0.7 & 0.3239 & 0.021 \\
$30^{\circ}$ Bevel & 0.7 & 0.507 & 0.010 \\
blunt & 0.7 & 1.169 & 0.045 \\
$10^{\circ}$ bevel & 0.7 & 0.2312 & 0.027 \\
$30^{\circ}$ bevel & 0.7 & 0.2438 & 0.012 \\
$45^{\circ}$ bevel & 0.7 & 0.4491 & 0.011 \\
$60^{\circ}$ bevel & 0.7 & 0.5132 & \\
\hline
\end{tabular}

The needle tip type factor is used to be an illustrative example to show the analysis of variance

(Table 2). In deformation phase, the blunt needle tip has the largest stiffness force, while the conical needle tip is diametrical (Fig. 6(c), $p=1.0289 \mathrm{E}-137, p<0.001$ ). In insertion phase, the conical tip has the highest insertion force growth rate, while the blunt tip is distinct. In addition, the conical and triangular tips which are symmetrical show a steady increase in insertion force, but the beveled and blunt tips which 
are asymmetrical exhibited periodic fluctuation. Moreover, microscopic observations using SZX7 series of stereoscopic microscope are carried out to investigate the interactions at needle tip and effects on insertion force. The triangular pyramid tip creates triangular shaped crack, blunt needle generates a ring crack and conical needle and bevel needle initiates a planar crack (Fig. 6(d)). It can be seen that the damage degree of tissue caused by conical needle is minimum while the blunt needle is maximum, and it goes conversely with the value of total insertion force.

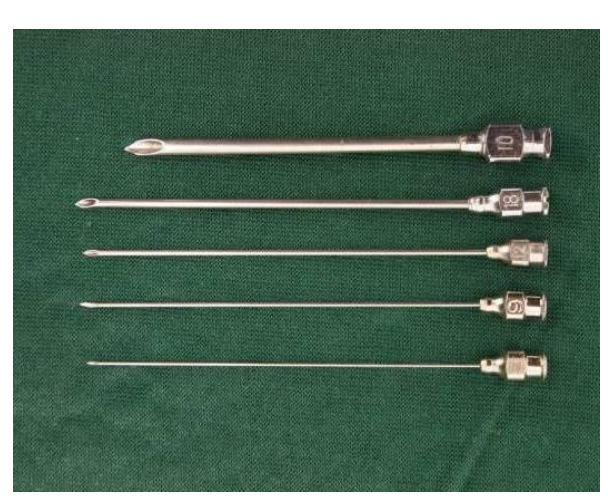

(a)
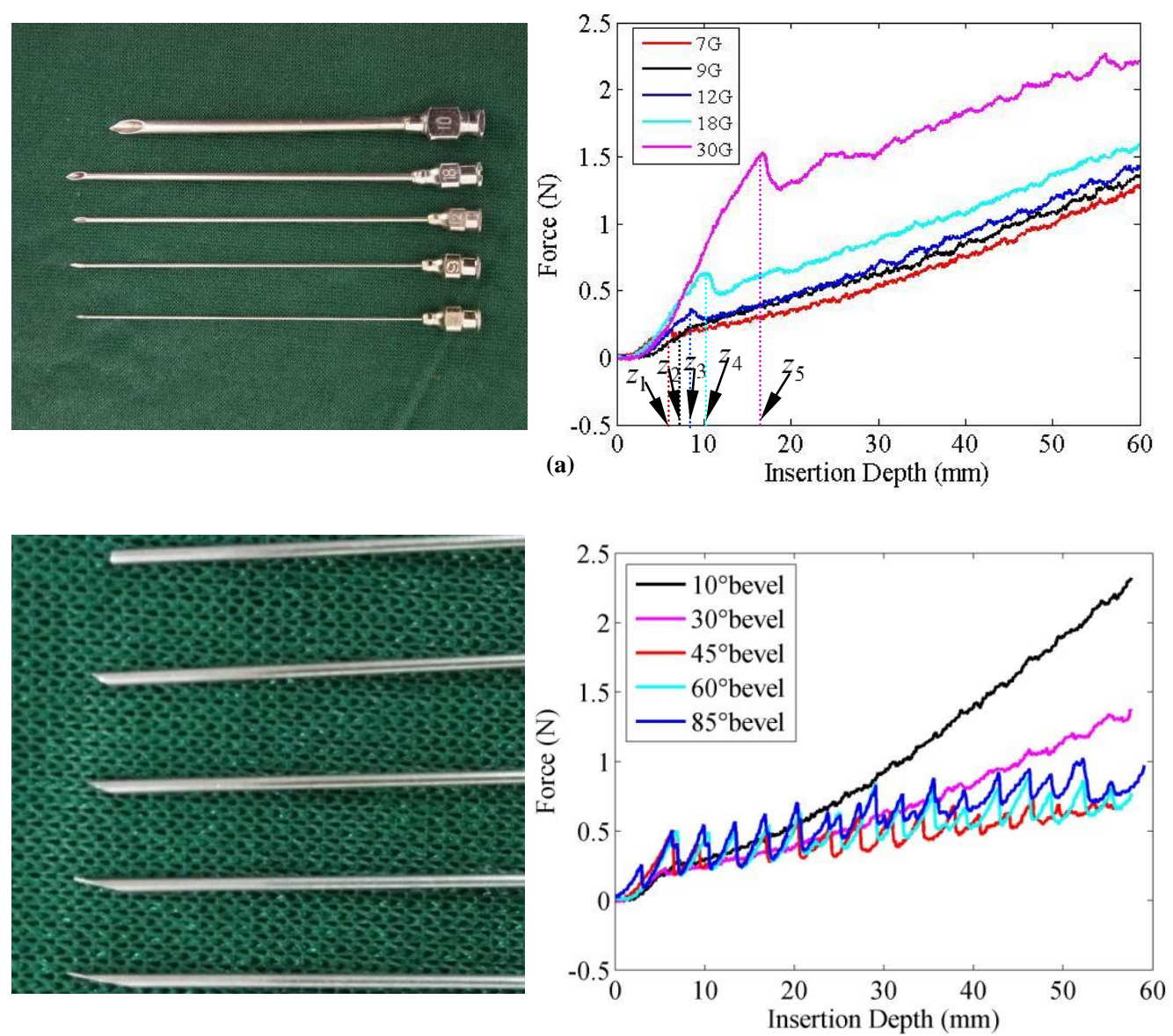

(b)
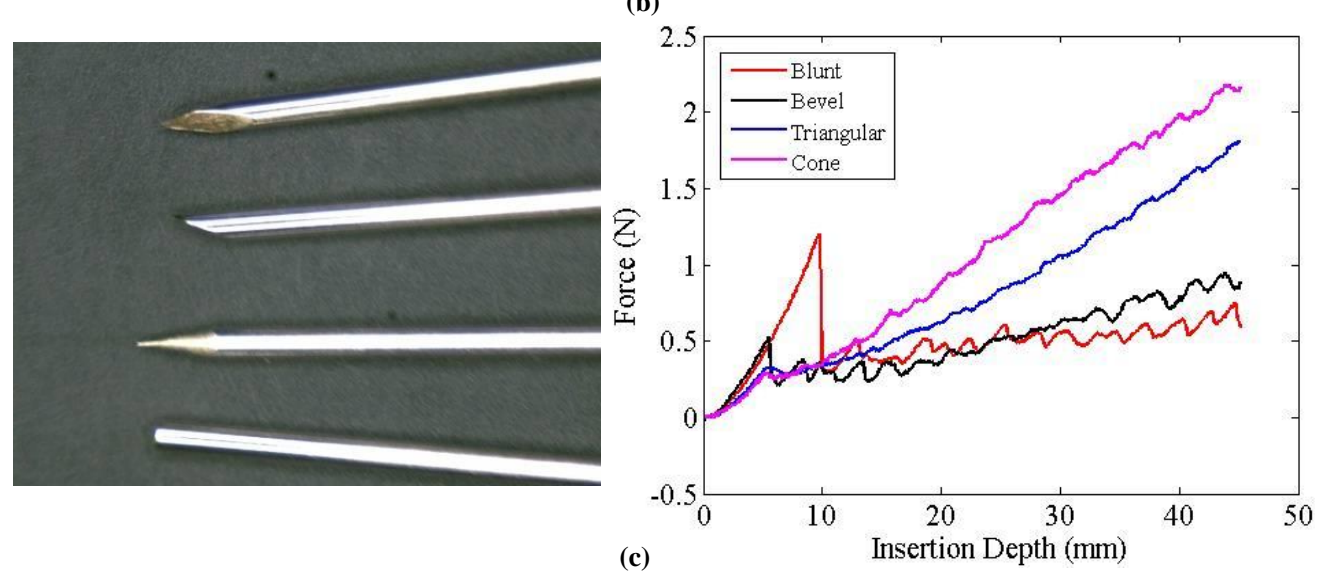


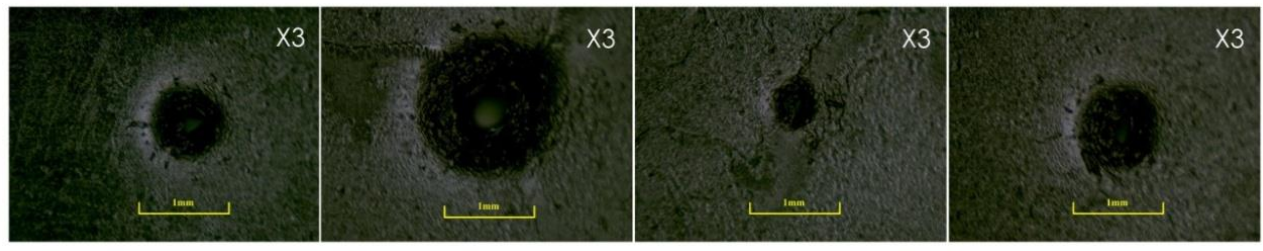

(d)

Fig. 6. The insertion forces versus displacement during insertion with different needle geometries into PVA phantom at $4 \mathrm{~mm} / \mathrm{s}$. (a) Diameter, (b) Bevel angle, and (c) Tip shape. (d) Crack shapes in PVA phantom. Left-to-right: triangular tip, blunt, conical, bevel angle.

Table 2

Analysis of variance table for illustrative example.

\begin{tabular}{|c|c|c|c|c|c|}
\hline Source of variation & df & SS & MS & $\mathrm{F}$ & $P$ \\
\hline Between treatments & 3 & 140.786 & 46.929 & 238.122 & $1.0289 \mathrm{E}-137$ \\
\hline Error (within treatments) & 2820 & 555.760 & 0.197 & & \\
\hline Total & 2823 & 696.546 & & & \\
\hline
\end{tabular}

df, degrees of freedom; F, test statistic; MS, mean squares; SS, sums of squares.

\subsection{Influence of tissue characteristics}

To investigate the influence of tissue characteristics on penetration, different biological tissues are used to substitute PVA phantoms. Needle-tissue interaction forces are depicted in detail, including the porcine skin (samples $n=5$, Fig. 7(a)), muscle and fat ( $\mathrm{n}=5$, Fig. $7(\mathrm{~b}), p \approx 0)$, liver capsule and vessel ( $\mathrm{n}=5$, Fig. $7(\mathrm{c}), p \approx 0)$. Results with skin present two peaks of force curve, which stand for the peak force of stratum corneum and dermis, respectively (Jee and Komvopoulos 2014). It can be seen that the peak force of dermis is 1.6 times larger than that of stratum corneum, with the same result of literature shown by Frick et al. (2001). And it suffered a sharp decrease before it reached an equilibrium 
$(0.5136 \mathrm{~N}$ with a fluctuation of $\pm 0.0356 \mathrm{~N})$, which is the same as literature shown by Butz et al. (2012) owing to the penetration from dermis to hypodermis. Experimental results suggest that insertion forces are growing linearly and the peak force of muscle and fat are $0.6078 \mathrm{~N}$ and $0.2040 \mathrm{~N}$, respectively. For the capsule and vessel of porcine liver, the stiffness forces are equal to $0.1623 \mathrm{~N}$ and $0.5912 \mathrm{~N}$ and the corresponding deformations are $11.74 \mathrm{~mm}$ and $7.456 \mathrm{~mm}$, respectively. It is obvious that the insertion force of skin and muscle is larger than fat, and probably it triples the stiffness peak force of fat. The experimental force data can be used to identify the location of needle tip and tissue-type.
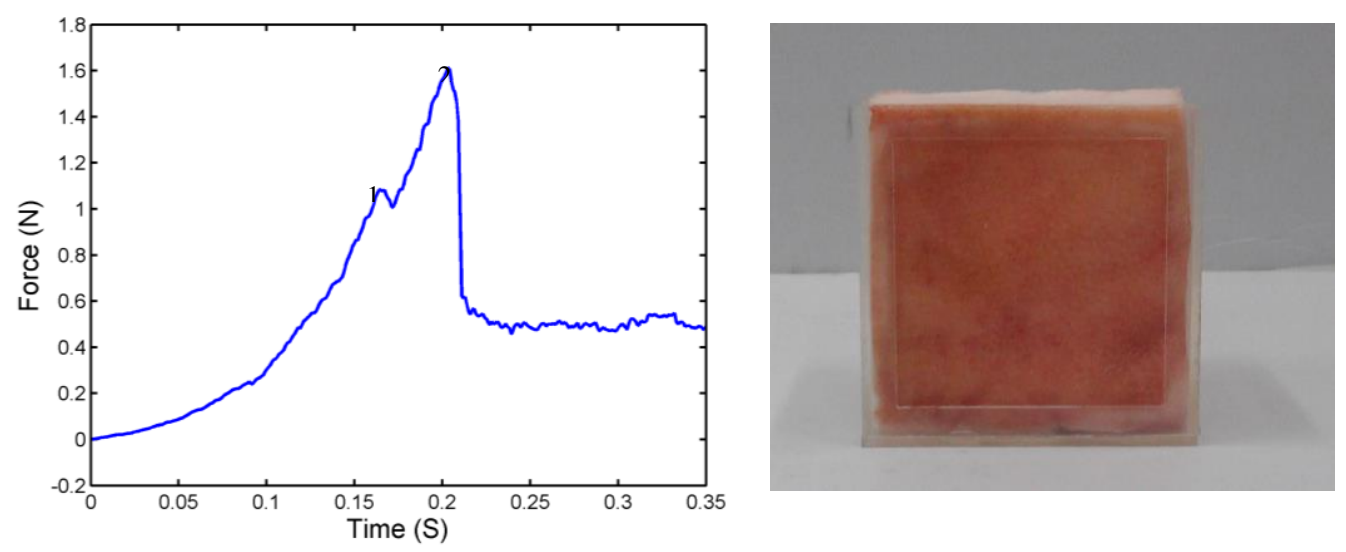

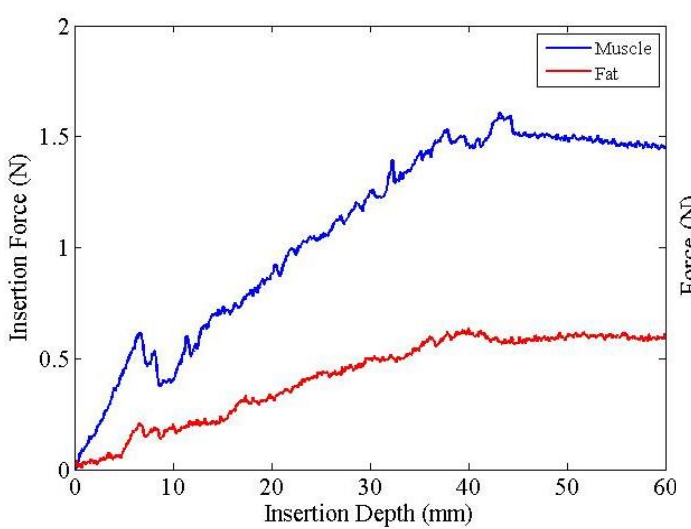

(b)

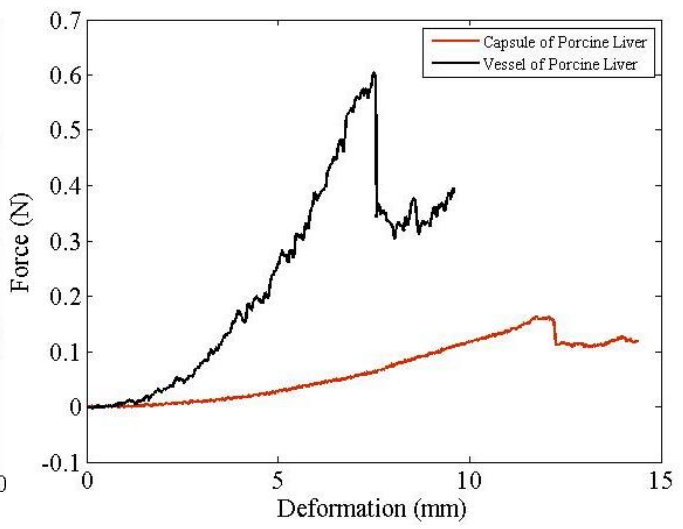

(c)

Fig. 7. The insertion forces of different tissues. (a) Skin, (b) Muscle and Fat, (c) Capsule and Vessel.

To make sure the influence of liver structure on insertion force, 5 samples of porcine liver were used to perform the experiment. Force curve profiles of penetrations into porcine liver can be seen in Fig.8 $(p=1.2386 \mathrm{E}-98, p<0.001)$. No obvious fluctuation appeared in penetration process, which 
indicates that vital vessels were inexistent in insertion trajectory. Whereas, there are two evident peak forces in position A and B, and the increment is larger than $0.6 \mathrm{~N}$. A precipitate drop appears in position C, which dues to the pass-through from vascular wall to vascular cavity until to vascular wall. There is a precipitate drop in position D but the variation is small, which is because of the asymmetry of tissue density. Force in position $\mathrm{E}$ has much bigger fluctuation frequency due to the insertion into vascular intensive areas.

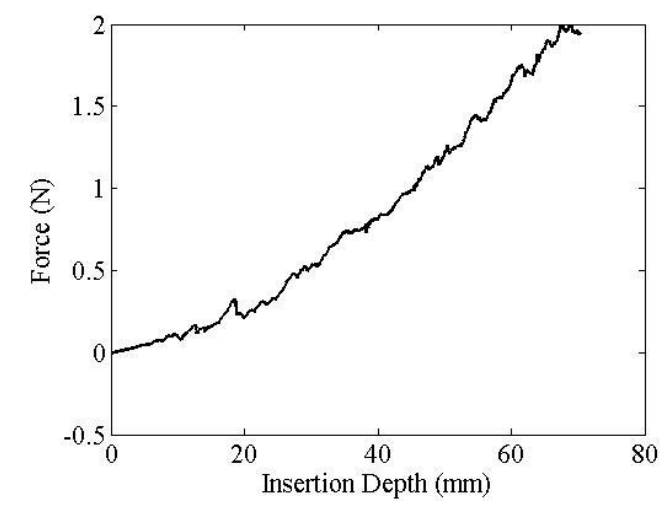

(a)

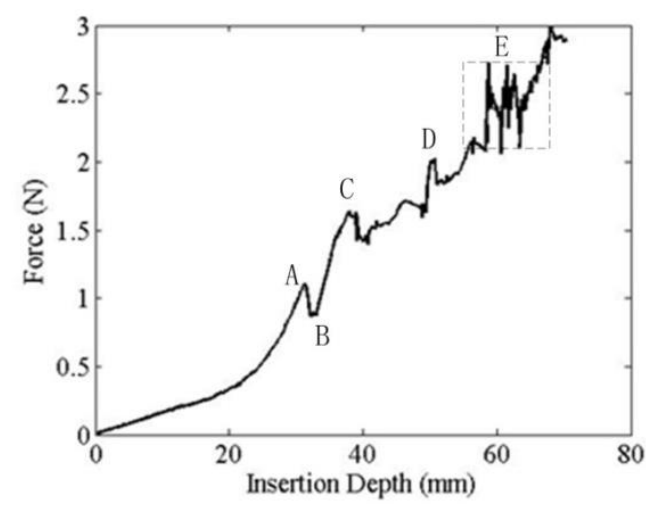

(b)

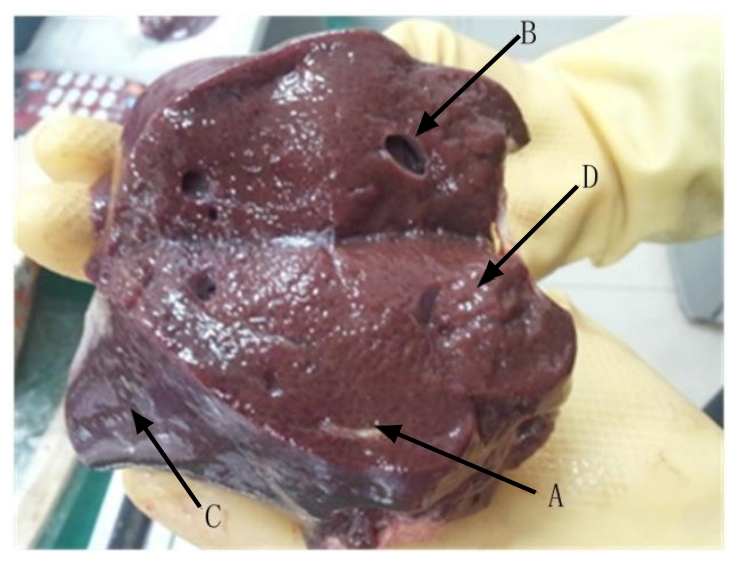

(c)

Fig. 8. The insertion force curves of twice insertions into porcine liver. (a) Safe insertion into porcine liver without meeting vital tissues, (b) Dangerous insertion into porcine liver, meeting some vital tissues, and (c) is a porcine liver, which is less than eight hours in vitro. A and B represent fascia and aortic endothelial vessel, while C and D stand for smooth surface and cross section, respectively. 
To imitate the intraoperative insertion process, 5 groups of multi-layer pork and PVA phantoms were used as materials in test. Each group of PVA phantoms was prepared with the thickness of 20mm, $20 \mathrm{~mm}$ and $25 \mathrm{~mm}$, which was used to simulate the fat, muscle and target, respectively. The insertion force curve of needle penetration through several tissue layers is shown in Fig.9 ( $p=2.6239 \mathrm{E}-136$, $p<0.001)$. Three rupture events occurred in penetration process, which can be inferred that a sharp force increase emerged when needle started to insert into another tissue. Stiffness force was nonexistent in Fig. 9(b), since the multi-layer pork was peeled and there was no large deformation. Sudden enlargement of insertion force indicates that the needle punctures into new organization structure.
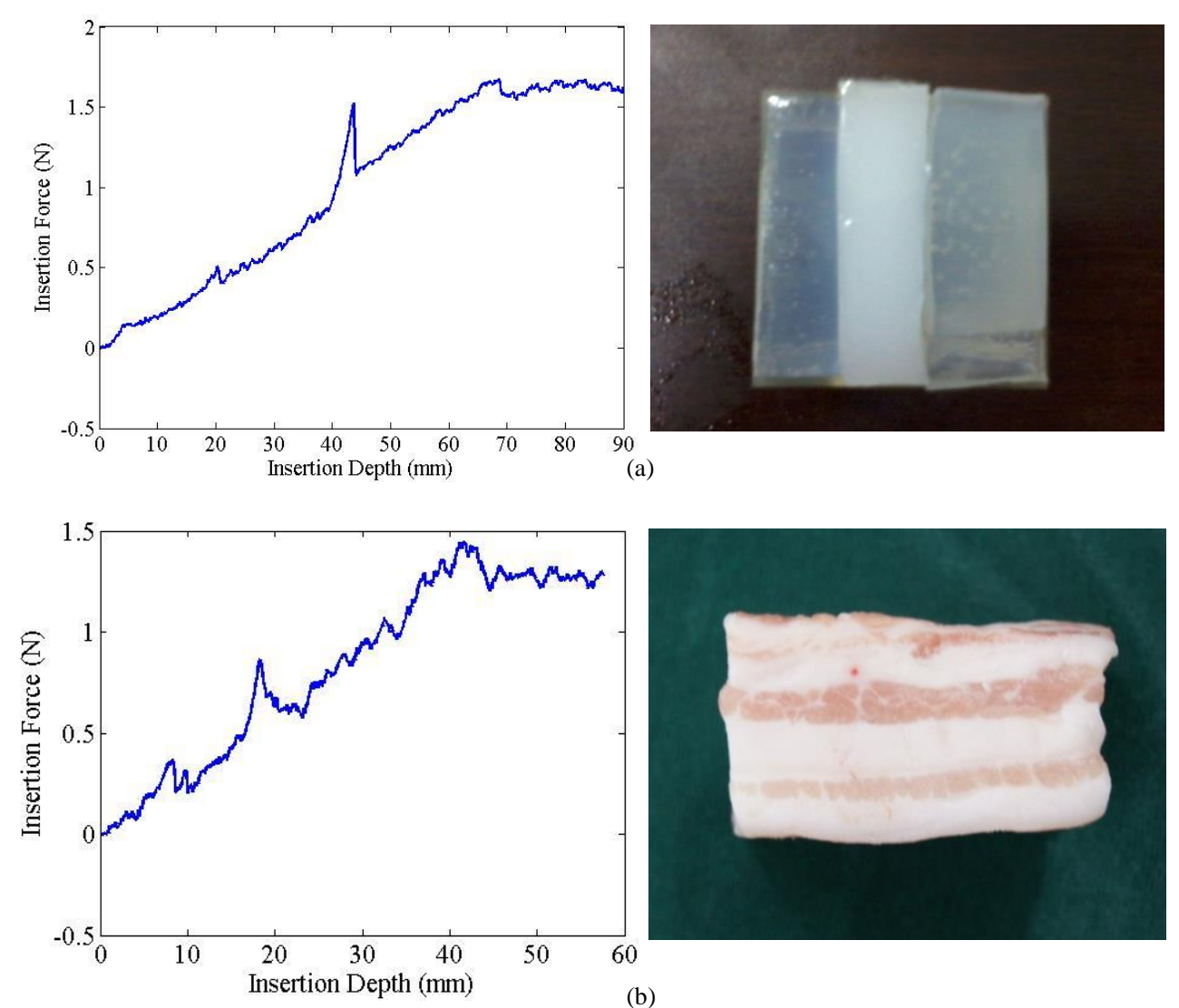

Fig. 9. Insertion forces acting on the needle when inserted through three layers that have different mechanical properties. (a) PVA phantom, (b) biological tissue.

\subsection{Comparisons with experimental results}


From Eq. (8) (13) and Fig. 6(a) it is clear that the stiffness force and friction force are increasing with diameter. Therefore the experimental insertion data obtained from different needle diameter is consistent with the result obtained from theoretical analysis. Figure 6(b) is found to be consistent with Eq. (5), because the stiffness force both enlarged with the increasing of bevel angle. The tissue density of fat is much less than muscle, that is $E_{2 f a t}<E_{2 m u s c l e}$. In addition, fat has an effect on lubrication, which reduces the friction coefficient between tissue and needle $\left(\mu_{f a t}<\mu_{\text {muscle }}\right)$. Seen by Eq. (13), the insertion force of fat is much less than muscle which is consistent with the experimental data. In general, the experimental result is basically coincident with the theory analysis expect for some slight data fluctuation, which may be caused by the insertion method.

\section{Discussion}

During the experimental process the interaction between needle and tissue is found to be influenced by the way the needle is inserted. For example, manual insertion will yield different results than automated insertion. Likewise, interrupted insertion results will be different from the continuous insertion, and force may be influenced by insertion velocity.

As illustrated in Fig.10, three groups of experiments are performed using a $7 \mathrm{G}$ bevel needle and PVA phantoms ( $\mathrm{n}=5$ for each group) with different insertion velocities, drive mode, and insertion processes. Experimental results show an increase slope of the force-position curve with the increasing of insertion velocity, which is from $0.5 \mathrm{~mm} / \mathrm{s}$ to $20 \mathrm{~mm} / \mathrm{s}$ (Fig. 10(a), $p=3.9871 \mathrm{E}-129, p<0.001$ ). Exponential growth appears initially, but total insertion force starts to remain constant when speed is up to $5 \mathrm{~mm} / \mathrm{s}$ or higher than that. However, the stiffness force seems to be independent of insertion velocity.

A contrast experiment between robot-assisted insertion and hand-held insertion is performed, and 3D Guidance track STAR electromagnetic locator was used to measure hand-held insertion velocity. 
Robot-assisted needle insertion has the similar stiffness force with manual insertion in deformation phase, while it has larger slope of force curve and less fluctuation in insertion phase. Hand-held insertion is unsteady yet, but it fluctuates around $5 \mathrm{~mm} / \mathrm{s}$ (Fig. 10(b), $p \approx 0)$.
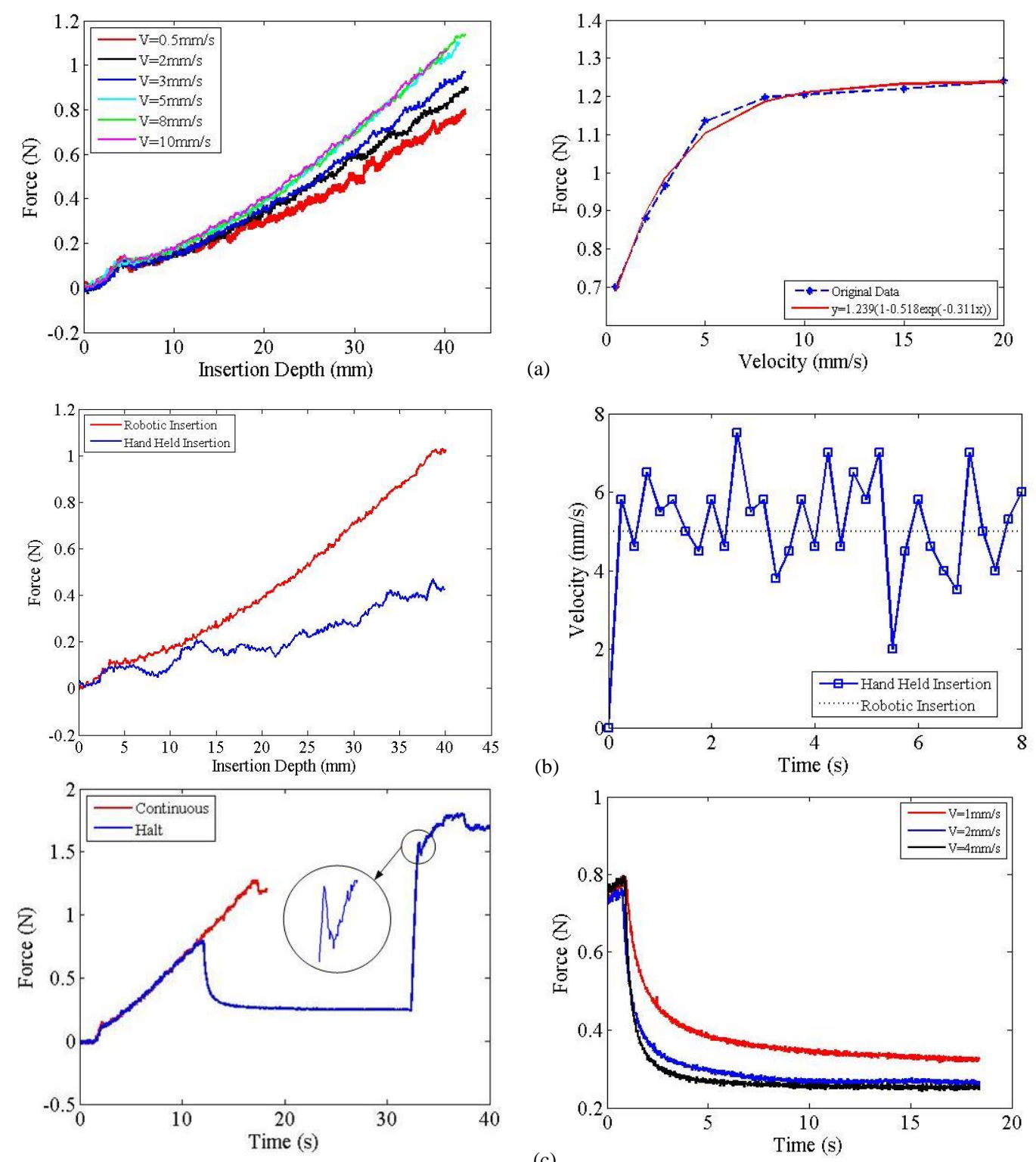

Fig. 10. The insertion forces versus displacement or times during insertion with different insertion methods into PVA phantom. 
(a) Insertion velocity, (b) Hand-held insertion vs. Robot-assisted insertion, and (c) Continuous insertion vs. interrupted insertion.

As we know, to ensure the precision of seed implantation, surgeons usually halt for several seconds in insertion process to search the target under image guided navigation. It indicates that the interrupted insertion has a higher maximum insertion force $(1.809 \mathrm{~N})$ and force density $(0.0282 \mathrm{~N} / \mathrm{mm})$ than that of continuous insertion $(1.278 \mathrm{~N}, 0.0196 \mathrm{~N} / \mathrm{mm}$ ) (Fig. 10(c), $p=2.8926 \mathrm{E}-156, p<0.001)$. An exponential decay in force was observed once the insertions halt, which is similar to the stiffness force in deformation phase. Obviously, the decay time of force is shorter when insertion velocity is faster, and the constant force is equal to $40 \%$ of the maximum force during interrupted insertion.

\section{Conclusion}

A systematic and experimental research was presented on needle-tissue interaction mechanics. Experiments are conducted where the needle geometric properties, insertion methods and tissue characteristics are varied. Analysis results of effect factors are shown in Table 3, in which the positive correlation, negative correlation or inconclusive correlation are remarked between stiffness force, friction force, and total force. Experimental results indicate that increasing needle diameter and insertion velocity results in larger insertion force. Varying needle tip shapes demonstrates that blunt needle produces largest puncture force and conical needle generates largest force slope. Different needle bevel angles from $10^{\circ}$ to $85^{\circ}$ result in insertion force decrease and stiffness force increase monotonically. Halting for 20s during insertion will enlarge insertion force. Robot-assisted needle insertion has a larger force compared with hand-held insertion. In view of tissue characteristics, when needle inserts into multi-layer tissue with different mechanical properties, several stiffness forces will emerge in the force curve. Insertion force of skin and muscle is larger than fat, and probably it triples the stiffness peak force of fat. If vessels occur in insertion trajectory, evident force variation and fluctuation 
will appear, which will be harmful to vital tissues.

Table 3

Analysis results of influence factors and empirical value of each parameter.

\begin{tabular}{|c|c|c|c|c|c|}
\hline & & $f_{\text {stiffness }}$ & $f_{\text {friction }}+f_{\text {cutting }}$ & $f_{\text {total }}$ & Empirical value \\
\hline \multirow[t]{3}{*}{ Needle geometry } & Needle diameter & + & & + & $1.2 \sim 1.8 \mathrm{~mm}$ \\
\hline & Tip edges & - & + & + & Beveled \\
\hline & Bevel angle & + & - & - & $10^{\circ} \sim 30^{\circ}$ \\
\hline \multirow[t]{3}{*}{ Insertion method } & Insertion velocity & & + & + & $5 \sim 10 \mathrm{~mm} / \mathrm{s}$ \\
\hline & Drive mode & & + & + & Continuous \\
\hline & Insertion process & 0 & + & + & Robot-assisted \\
\hline Tissue & Muscle, fat vs. skin & + & + & + & Skin \\
\hline \multirow[t]{3}{*}{ characteristic } & Liver capsule and vessel & + & & + & Trajectory \\
\hline & Multi-layer phantom & & & & \\
\hline & Multi-layer tissue & & & & \\
\hline
\end{tabular}

+ , pos. correlation; -, neg. correlation; 0 , no corr. or inconclusive.

Influence factors presented in this study can be considered and added to construct more accurate needle-tissue interaction force models in future. The research on needle geometries can assist to optimize needle design (Groves et al. 2012) by using suitable diameter, needle tip, and bevel angle (see Table 3). The study of insertion methods is beneficial for robotically steering needles by means of choosing appropriate insertion velocity and operation strategy. The research on tissue characteristics could be favorable for providing the location of needle tip during penetration, which can be seen from 
the trend of force curve mentioned in Section 3. In this paper, the research was focused on discussing single influence factor, while future work will be extended to multivariate analysis, and the effects of variable parameters to needle deflection should be investigated.

\section{Conflict of interest statement}

Authors declare no conflict of interest regarding the submitted manuscript.

\section{Acknowledgments}

We gratefully acknowledge financial support from the National Natural Science Foundation of People's Republic of China (51175373), and New Century Educational Talents Plan of Chinese Education Ministry (NCET-10-0625).

\section{References}

Abolhassani, N., Patel, R., and Moallem, M., 2007. Needle insertion into soft tissue: A survey. Medical Engineering \& Physics 29, 413-431

Biot, M.A., 1937. Bending of an infinite beam on an elastic foundation. Journal of Applied Mechanics 59, A1-7.

Butz, K.D., Griebel, A.J., Novak, T., Harris, K., Kornokovich, A., Chiappetta, M.F., Neu, C.P., 2012. Prestress as an optimal biomechanical parameter for needle penetration. Journal of Biomechanics 45, 1176-1179.

Choy, Y.B., Cao, H., Tungjitkusolmun, S., Tsai, J.Z., Haemmerich, D., Vorperian, V.R., et al., 2002. Mechanical compliance of the endocardium. Journal of Biomechanics 35, 1671-1676.

DiMaio, S.P., and Salcudean, S.E., 2005. Interactive simulation of needle insertion models. IEEE Transactions on Biomedical Engineering 52, 1167-1179.

DiMaio, S.P., and Salcudean, S.E., 2003. Needle insertion modeling and simulation. IEEE Transactions on Robotic and Automation 19, 864-875.

Frick, T.B., Marucci, D.D., Cartmill, J.A., Martin, C.J., and Walsh, W.R., 2001. Resistance forces acting on suture 
needles. Journal of Biomechanics 34, 1335-1340.

Gerwen, D.J., Dankelman, J., and Dobbelsteen, J.J., 2012. Needle-tissue interaction forces-A survey of experimental data. Medical Engineering \& Physics 34, 665-680.

Groves, R.B., Coulman, S.A., Birchall, J.C., and Evans, S.L., 2012. Quantifying the mechanical properties of human skin to optimise future microneedle device design. Computer methods in biomechanics and biomedical engineering 15, 73-82.

Hing, J.T., Brooks, A.D., and Desai, J.P., 2006. Reality-based needle insertion simulation for haptic feedback in prostate brachytherapy. In: Proceeding of IEEE international conference on robotics and automation, IEEE, pp. $619-624$.

Jee, T., Komvopoulos, K., 2014. Skin viscoelasticity studied in vitro by microprobe-based techniques. Journal of Biomechanics 47, 553-559.

Jiang, S., Liu, S., and Feng, W.H., 2011. PVA hydrogel propertied for biomedical application. Journal of the Mechanical Behavior of Biomedical Materials 4, 1228-1233.

Lewis, M.C., Lafferty, J.P., Sacks, M.S., Pallares, V.S., and TerRiet, M., 2000. How much work is required to puncture dura with tuohy needles? British Journal of Anaesthesia 85, 238-241.

Mahvash, M., and Dupont, P.E., 2010. Mechanics of dynamic needle insertion into a biological material. IEEE Transactions on Biomedical Engineering 57, 934-943.

Meltsner, M.A., Ferrier, N.J., and Thomadsen, B.R., 2007. Observations on rotating needle insertions using a brachytherapy robot. Physics in medicine and biology 52, 6027-6037.

Misra, S., Reed, K.B., Schafer, B.W., Ramesh, K.T., and Okamura, A.M., 2010. Mechanics of flexible needles robotically steered through soft tissue. The International Journal of Robotics Research 29, 1640-1660.

Okamura, A.M., Simone, C., and O’Leary, M.D., 2004. Force modeling for needle insertion into soft tissue. IEEE 
Transactions on Biomedical Engineering 51, 1707-1716.

Sneddon, L.N., 1965. The relation between load and penetration in the axisymmetric boussinesq problem for a punch of arbitrary profile. International Journal of Engineering Science 3, 47-57.

Stellman, J.T., 2009. Development, production, and characterization of plastic hypodermic needles. MSc. Thesis, Georgia Institute of Technology.

Yan, K.G., Podder, T., Xiao, D., Yu, Y., Liu, T.I., Cheng, C., Ng, W.S., 2006. An improved needle steering model with online parameter estimator. International Journal of Computer Assisted Radiology and Surgery, 205-212.

Yankelevsky, D.Z., Eisenberger, M. and Adin, M.A., 1989. Analysis of beams on nonlinear winkler foundation. Computers \& Structures 31, 287-292. 